\title{
Oriented Texture Completion by AM-FM Reaction-Diffusion
}

\author{
Scott T. Acton, Senior Member, IEEE, Dipti Prasad Mukherjee, Joebob P. Havlicek, Senior Member, IEEE, and \\ Alan Conrad Bovik, Fellow, IEEE
}

\begin{abstract}
We provide an automated method to repair broken, occluded oriented image textures. Our approach is based on partial differential equations (PDEs) and AM-FM image modeling. Reconstruction of the texture occurs via simultaneous PDE-generated diffusion and reaction. In the diffusion process, the image is adaptively smoothed, preserving important boundaries and features. The reaction process produces the reconstructed textural information in the occluded image regions. Gabor filters are designed and used in the reaction process using an AM-FM dominant component analysis. An AM-FM model of the texture image is constructed, making it possible to localize the reaction filters spatio-spectrally. In contrast to previous disocclusion techniques that depend on interpolation, on continuity of the connected components within the image level sets, or on texture estimation, the reaction-diffusion process proposed here yields a seamless transition between the recreated region and the unoccluded image regions. Using AM-FM dominant component analysis, we avoid the ad hoc parameter selection typified with other reaction-diffusion approaches. As a useful example, we focus on the repair of broken, occluded fingerprints. We also treat several exemplary natural textures to demonstrate the technique's generality.
\end{abstract}

Index Terms-AM-FM image models, anisotropic diffusion, disocclusion, texture.

\section{INTRODUCTION}

$\mathbf{R}$ EPAIR of occluded or missing parts of digital images is an important problem that has been studied by psychophysicists [18], mathematicians [29], computer scientists and signal processing engineers [31]. The texture completion or disocclusion problem can be solved adequately for small occlusions by forcing continuity of image intensity and edges [29], [31]. When large occlusions or substantial missing portions of the image exist, it is likely that continuation-based methods will fail, since internal variations due to patterns and detail exist within the missing region.

This paper focuses on the reconstruction of large missing regions of homogeneous oriented textures. To reconstruct these

Manuscript received January 31, 2000; revised February 19, 2001. The associate editor coordinating the review of this manuscript and approving it for publication was Prof. Patrick Bouthemy.

S. T. Acton is with the Department of Electrical and Computer Engineering, University of Virginia, Charlottesville, VA 22902 USA (e-mail: acton@virginia.edu).

D. P. Mukherjee is with the Electronics and Communication Sciences Unit, Indian Statistical Institute, Calcutta, India 700035.

J. P. Havlicek is with the School of Electrical and Computer Engineering, University of Oklahoma, Norman, OK 73069 USA (e-mail: joebob@ou.edu).

A. C. Bovik is with the Laboratory for Image and Video Engineering, Center for Vision and Image Sciences, Department of Electrical and Computer Engineering, University of Texas at Austin, Austin, TX 78712-1084 USA.

Publisher Item Identifier S 1057-7149(01)04473-6. textures, two processes must be considered. First, a suitable texture must be generated that matches that in the image. Second, the texture must be adapted to the missing region so that the human observer may perceive a seamless texture image.

In our approach, we provide a robust method of pattern estimation and generation. The input image is modeled within an AM-FM framework, and the dominant components of the oriented texture are estimated at each position. Dominant image components in a region surrounding the occlusion are used to generate texture for the region of interest. In contrast to methods that generate a texture and attempt to insert this texture within the image in one step, our approach adapts or grows the texture via partial differential equations (PDEs). A reaction-diffusion mechanism, in the spirit of Turing's morphogenesis [38], is applied. Here, texture generation and smoothing are combined using coupled PDEs. The reaction mechanism utilizes the AM-FM dominant component analysis to enforce a suitable pattern on the missing region. At the same time, anisotropic diffusion is used to adaptively smooth the image, producing a seamless restoration.

As a significant and useful example, we apply the methods we develop to the problem of fingerprint repair. Often, regions within fingerprint images are lost due to the inhomogeneity of the surface, movement of the finger (smudging), partial contact, or problems associated with imaging the fingerprint. The generation of fingerprint-like textures is well established [19], [37]. Typically, a bank of Gabor filters is used to replicate the undulating patterns observed in fingerprints. The selection of the particular Gabor filters is accomplished using a generalized model [19] or by trial and error. To demonstrate generality of this approach, we also apply the method to several other naturally occurring textural regions.

In the following section, we discuss relevant advances in pattern generation and enhancement, and we also review work in image disocclusion. The reconstruction of image textures begins with the reaction-diffusion model outlined in Section III. The success of reaction-diffusion for disocclusion is based on the AM-FM dominant component analysis described in Section IV. Results for the proposed method and other existing methods are given in Section V followed by conclusions.

\section{BACKGROUND}

Reaction-diffusion equations have been employed to simulate patterns abundant in nature. For example, a key issue in developmental biology is the dynamic arrangement of embryonic cells into particular patterns. Turing [38] suggested that two or more chemicals can diffuse and react within neighboring cells, 


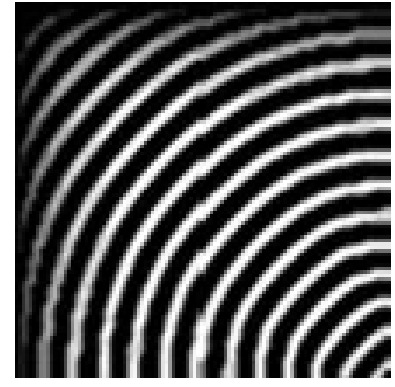

Fig. 1. Stripe formation using coupled PDE.

depending on the concentration of the cell and its neighborhood. He proposed a set of coupled PDEs to simulate such patterns, $(\delta a / \delta t)=F(a, b)+D_{a} \nabla^{2} a$, and $(\delta b / \delta t)=G(a, b)+$ $D_{b} \nabla^{2} b$. In this case, $a$ and $b$ are the two chemicals that diffuse depending on neighborhood concentration (computed via the Laplacian operator), and $D_{a}$ and $D_{b}$ are the corresponding diffusion rate constants. Changes of $a$ and $b$ per time unit are given by $\delta a / \delta t$ and $\delta b / \delta t$ respectively. The local concentrations $F(a, b)$ and $G(a, b)$ are iteratively computed, and the process of reaction-diffusion continues until a stable pattern emerges.

The Turing model was subsequently extended by Meinhardt [30] to simulate the stripe formation process. An intuitive understanding of this technique could be obtained if we consider a situation where concentration and diffusion of chemical $a$ prohibits chemical $b$ to be present at the same place and at the same time. So, the chemicals are locally exclusive. A striped pattern generated by the PDE model in [30] is shown in Fig. 1. Similar models have been used to produce a variety of synthetic textures [40]. A major difficulty of this approach is the selection of the constants needed to generate a stable pattern. Price $e t$ al. [35] have used similar coupled PDE models to enhance fingerprint images and addressed the issue of parameter selection. For the texture completion problem, generating patterns of a specific granularity and directionality is difficult, as is matching the pattern at the boundary of the occluded region. Thus, pattern formation is possible with the Meinhardt approach, but an acceptable disocclusion solution is not amenable. In a related PDE based application to supervised texture segmentation, Paragios and Deriche [32] have used a global statistical texture model. The contour detection for a homogeneous pixel cluster and region based segmentation are integrated in a single framework defining a geodesic active region.

In a similar context, Sherstinsky and Picard [37] have introduced the $M$-lattice system to produce restored textures from corrupted imagery. The $M$-lattice is a nonlinear dynamic system founded on reaction-diffusion. In the $M$-lattice system, a warping function is introduced in the reaction process to facilitate stability. These warping functions are typically sigmoidal functions that prevent numerical overflow at every time step. The $M$-lattice system uses orientation sensitive filters, similar to the flow field analysis in [19].

Zhu et al. [42] have proposed a statistical theory for texture modeling with the objective of texture synthesis. A set of filters is selected from a general filter bank to capture texture features. The histograms of the filtered images estimate a marginal distribution of the image. A maximum entropy distribution based

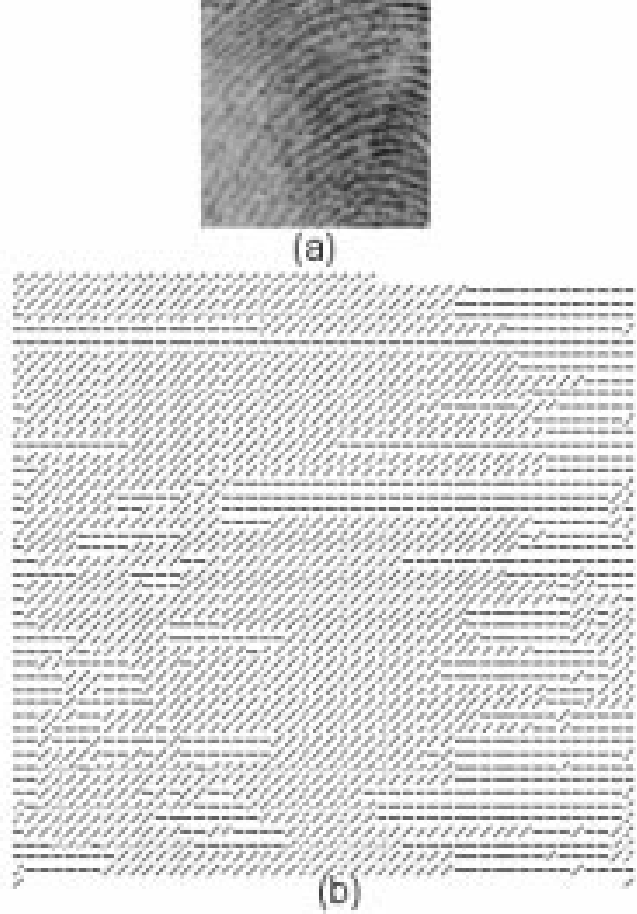

Fig. 2. (a) Original "oriented" fingerprint image. (b) Flow field depicting orientation of patterns of Fig. 2(a). For visual clarity, the flow field orientations are plotted in a magnified scale and quantized according to the following orientation ranges: $(0, \pi / 4),(\pi / 4, \pi / 2),(\pi / 2,3 \pi / 4)$, and $(3 \pi / 4, \pi)$.

technique is used to fuse these features to generate a unified texture model for texture synthesis. By contrast, our texture model is based on AM-FM modeling and dominant component analysis, which are most suitable for locally narrow-band, quasi-periodic repetitive patterns. Nonparametric statistical sampling is also used by Efros et al. [7] for texture synthesis.

Kass and Witkin [19] have also investigated the generation of oriented textures. Since the pattern embedded in a fingerprint essentially consists of oriented contours, their flow field analysis model is suitable for fingerprint pattern generation and analysis. Bandpass filters, similar to those of the classical Marr-Hildreth scheme [28], are used for edge detection. The Kass/Witkin method provides a flow field with direction vectors at every point of the oriented pattern. The flow field for the "oriented" fingerprint image in Fig. 2(a) is shown in Fig. 2(b). The flow field orientations are plotted in a magnified scale and quantized by the following set of four orientations: $(0, \pi / 4),(\pi / 4, \pi / 2)$, $(\pi / 2,3 \pi / 4)$, and $(3 \pi / 4, \pi)$. In [19], it is suggested that this method could be used to synthesize fingerprints under limited occlusion as they become extremely regular in flow field coordinates.

For the general problem of image disocclusion, Masnou and Morel [29] have proposed a solution that exploits the connected components within the image level sets. The level set $l$ at the intensity $g$ of an image $I$ is given by the set $l=\{x: I(x) \geq g\}$ where $I(x)$ is the intensity of the image at location $x$. Level lines are then defined as the boundaries of connected components within the image level sets. In an occluded area, Masnou and Morel enforce continuity of the level lines to reconstruct the occluded region. A cost function is used to minimize the total variation in angle for the connected pairs of level line termina- 


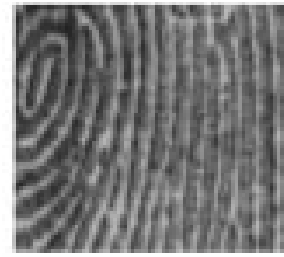

(a)

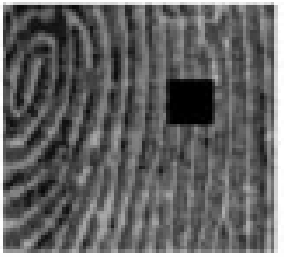

(b)

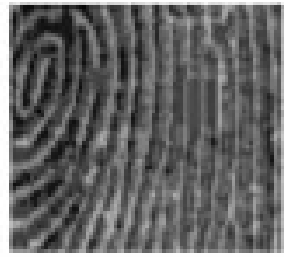

(c)
Fig. 3. (a) Original "stripe" fingerprint image. (b) After occlusion (square region in black) in Fig. 3(a). (c) After disocclusion of Fig. 3(b) following the level line method.

tions. A term within the cost function enforces continuity of the level lines. A simplified version of this method (using only horizontal and vertical level lines) is implemented for the original "stripe" fingerprint image as shown in Fig. 3(a). The result of disocclusion through continuity of level sets in the square occluded region of Fig. 3(b) is shown in Fig. 3(c). For small occlusions, this method proves to be effective. However, for larger occlusions where the pattern curves within the occluded area, the level line continuation method is unsuccessful. The level set method produces piecewise constant stripes in the repaired fingerprint, which appear unnatural in the reconstruction [see Fig. 3(c)]. The same criticism could be levied on the classical interpolation-based disocclusion solutions [31]. Therefore, an adaptive pattern formation technique is required for the fingerprint latency problem.

Kokaram et al. [20]-[23] have performed extensive studies on detecting and interpolating missing data in image sequences. Their work involves primarily two components: estimation of motion using MRF models for spatio-temporal changes in corresponding blocks of a movie image sequence and generation of a variety of interpolators including median, MRF and AR based techniques. The approach is most suitable for detecting and interpolating small homogeneous image mass that is uncorrelated with neighboring regions. For the generation of oriented texture features, which is the focus of this paper, domain specific heuristics and exploitation of characteristics specific to image sequences may not be appropriate.

Rather than manual selection of the pattern regenerating filters, our method hinges on an automated AM-FM dominant component analysis. Joint AM-FM modeling of one-dimensional (1-D) signals has recently been studied extensively [3], [4], [25]. In two-dimensional (2-D) images, the Teager-Kaiser operator [26], [27] and other related techniques [2], [9], [15] have been used to extract dominant AM-FM information from oriented textured images. Multicomponent multidimensional AM-FM models have also been investigated recently [13], [15]. Here, we employ dominant component analysis in the design of reaction filters that are used in a reaction-diffusion process. In Section III, we describe the reaction-diffusion mechanism. Section IV details the dominant component analysis.

\section{REACTION-DIFFUSION FOR TEXTURE COMPLETION}

Zhu and Mumford [41] have motivated the use of reaction-diffusion models in image processing with an analysis of universal image statistics. Analyzing a set of natural images, they have shown that a family of potential functions can be used in a reaction-diffusion paradigm to capture the smoothness and also the prominent patterns of an image. The typical potential functions lead to image smoothing via anisotropic diffusion. Inverted potential functions produce pattern formation or reaction. From this basis, we approach the problem of texture disocclusion.

The reaction-diffusion mechanism used for texture disocclusion is

$$
\frac{\partial \mathbf{I}}{\partial t}=\rho_{\mathrm{D}} \mathbf{D}+\rho_{\mathrm{R}} \mathbf{R}
$$

where $\mathbf{D}$ is the diffusion term, $\mathbf{R}$ is the reaction term; and $\rho_{\mathrm{D}}$ and $\rho_{\mathrm{R}}$ are the rate of diffusion and reaction, respectively. For a specific image location $\mathrm{x}=(x, y)$, we have

$$
\frac{\partial I(\mathbf{x})}{\partial t}=\rho_{\mathrm{D}}(\mathbf{x}) D(\mathbf{x})+\rho_{\mathrm{R}}(\mathbf{x}) R(\mathbf{x}) .
$$

A discrete Jacobi update for (2) is given by

$$
I_{t+1}(\mathbf{x}) \leftarrow I_{t}(\mathbf{x})+\rho_{\mathrm{D}}(\mathbf{x}) D(\mathbf{x})+\rho_{\mathrm{R}}(\mathbf{x}) R(\mathbf{x})
$$

where $I_{t}(\mathrm{x})$ is the intensity of position $\mathrm{x}$ at iteration $t$ and $\mathrm{x} \in$ $\mathbf{Z}^{2}$. The initial image intensities in $\mathbf{I}_{0}$ are equal to those in the input image $\mathbf{I}$, except in the case of the occluded region.

For the occluded region, we "seed" the reaction-diffusion process with noise that is distributed identically to that of the surrounding region. Let $\Omega$ denote the domain of the image and $\mathbf{U} \subset \Omega$ denote the unoccluded region. Let $\mathbf{B} \subset \mathbf{U}$ denote the region surrounding the occlusion/latency and $\mathbf{O}=\mathbf{U}^{c}$ denote the occluded region. If $\mathbf{x} \in \mathbf{U}$, then $I_{0}(\mathbf{x})=I(\mathbf{x})$. But, if $\mathbf{x} \in \mathbf{O}$, then $I_{0}(\mathbf{x})=R$, where $R$ is a random variable with density $f_{R}(i)=H_{\mathbf{B}}(i) /|\mathbf{B}|$ where $H_{\mathbf{B}}(i)$ is the intensity histogram for region $\mathbf{B}$ and $|\mathbf{B}|$ is the cardinality of $\mathbf{B}$. The width of $\mathbf{B}$ (the region surrounding the occlusion) depends on, e.g., the maximum ridge-to-ridge spacing in a fingerprint pattern. Let $T_{\max }$ denote this maximum width (estimated by the AM-FM dominate component analysis presented in Section IV). Then, we define the width of $\mathbf{B}$ to be $2 T_{\max }$ pixels. Using this method of defining the boundary region $\mathbf{B}$, we ensure that the width of the boundary region exceeds one full pattern period.

Seeding the region with noise identically distributed as the intensities of the surrounding region has the effect of providing a disocclusion solution admitting intensity distribution and contrast similar to the image. If uniformly distributed noise is used instead, as was done in [41], the repaired region tends not to match the surrounding region in graylevel distribution. This typically results in an unnatural appearance.

In the texture disocclusion problem, several aspects of equation (2) are important. Since the disocclusion process not only generates a pattern but also adapts the pattern to the existing boundaries, the reaction-diffusion approach excels in mating the new pattern with the existing unoccluded pattern (as compared to nonadaptive texture generation approaches such as [30]). In the case of [30], the stripe generation process is independent of the orientation of the texture at boundaries of the occluded region. 


\section{A. Diffusion Model}

Given the basic reaction-diffusion model, we now define the diffusion and reaction terms for texture disocclusion. Diffusion and reaction have conflicting objectives. The goal of diffusion is smoothing, while the goal of reaction is pattern formation. Without diffusion, a smooth texture pattern could not be generated from the seed noise. Since anisotropic diffusion encourages intra-region, not inter-region, smoothing, the texture can be smoothed without eliminating the important intensity transitions (edges). A continuous anisotropic diffusion PDE [33] is

$$
\frac{\partial I_{t}(\mathbf{x})}{\partial t}=\operatorname{div}\left\{c(\mathbf{x}) \nabla I_{t}(\mathbf{x})\right\}
$$

where $c(\mathbf{x})$ is the diffusion coefficient. Alternatively, the diffusion operator can be expressed as a combination of $c_{\xi} I_{\xi \xi}+c_{\eta} I_{\eta \eta}$ where $I_{\xi \xi}$ and $I_{\eta \eta}$ represent second directional derivatives along image gradients and the normal respectively [24].

The equivalent discrete representation of (4) for substitution in (3) is given by

$$
D(\mathbf{x})=\sum_{d=1}^{\Gamma} c_{d}(\mathbf{x}) \nabla I_{d}(\mathbf{x})
$$

where $\Gamma$ is the number of directions in which diffusion is computed and $\nabla I_{d}(\mathbf{x})$ is the directional derivative (simple difference) in direction $d$ at location $\mathrm{x}$. For $\Gamma=4$, we use the simple differences $\nabla I_{d}(\mathrm{x})$ with respect to the "western," "eastern," "northern," and "southern" neighbors. For example, if $\mathrm{x}=(x, y), \nabla I_{1}(x, y)=I\left(x-h_{1}, y\right)-I(x, y)$. Here, the parameter $h_{1}$ defines the sample spacing used to estimate the directional derivative in the $d=1$ direction (and is typically unity-valued).

The selection of the diffusion coefficient $c(\mathbf{x})$ is the most important step in designing the diffusion process. Essentially, we want a diffusion coefficient that is low (near zero) at image edges and is high (near one) within image regions. With $k$ as an edge strength parameter, a logical choice is given by [33]

$$
c(\mathbf{x})=\exp \left\{-\left[\frac{\nabla I(\mathbf{x})}{k}\right]^{2}\right\} .
$$

With the initial solution for disocclusion seeded with noise, the traditional diffusion coefficients cannot remove significant outliers [where $|\nabla I(\mathrm{x})| \gg k$ ]. To regularize the diffusion operation, we use a modification of the gradient image used to compute the diffusion coefficients, as suggested by [5]. A Gaussianconvolved version of the image is then utilized in computing the gradient magnitudes used in the diffusion coefficients

$$
c(\mathrm{x})=\exp \left\{-\left[\frac{\nabla S(\mathrm{x})}{k}\right]^{2}\right\}
$$

where $\mathbf{S}=\mathbf{I}^{*} \mathbf{g}_{\sigma}$ is the convolution of $\mathbf{I}$ with a Gaussian of standard deviation $\sigma$. The regularized diffusion given by (7) is stable and well posed [5]. Another regularized implementation, called morphological anisotropic diffusion, can be formed by substituting $\mathbf{S}=(\mathbf{I} \circ \mathbf{E}) \bullet \mathbf{E}$ into (7) [36]. In this case, $\mathbf{E}$ is a structuring element of size $m \times m, \mathbf{I} \circ \mathbf{E}$ is the morphological opening of $\mathbf{I}$ by $\mathbf{E}$, and $\mathbf{I} \bullet \mathbf{E}$ is the morphological closing of I by $\mathbf{E}$. However, for texture disocclusion, the morphological approach tends to flatten image regions, leading to a piecewise constant result. The constant regions are not appropriate models for highly oriented, repetitive textures. On the other hand, the smooth Gaussian filter result does produce smooth transitions in image intensity within the texture.

In the above diffusion model using (7), there are two parameters: $\sigma$ and $k$. For the case of diffusion within the texture pattern, these diffusion parameters can be selected without ambiguity. First, since $\sigma$ controls the scale of the features retained in diffusion, the value of $\sigma$ is set to $\sigma=T_{\min }$, the minimum periodicity of the texture pattern (e.g., in a fingerprint, the minimum distance between ridges). We compute $\sigma$ using the dominant components extracted in the analysis of Section IV. Since it controls the maximum change between pixels, $k$ is set to $k=C_{\max }$, the maximum contrast (intensity difference) within the texture pattern in the surrounding area $\mathbf{B}$.

\section{B. Reaction Model}

In the reaction process, we encourage formation of patterns of a given granularity and directionality, corresponding to a localized area in the frequency domain covered by a specific Gabor filter $\mathbf{G}$ given by

$$
\mathbf{G}=\cos [(2 \pi / N)(u x+v y)] g_{\sigma}(x, y)
$$

for an $N \times N$ image indexed by $(x, y)$ and a Gaussian $g_{\sigma}(x, y)$, where $\sigma$ is the scale parameter (standard deviation of the Gaussian). In this case, the Gabor function has standard deviation (width) of $\sigma$ and center frequency $u, v$. The Gabor parameters are automatically determined by the AM-FM dominant component analysis method given in Section IV.

To produce patterns that correspond to oriented texture features, the reaction term is given by

$$
R(\mathbf{x})=\mathbf{G}_{\mathbf{x}} \otimes\left[\varphi\left(\mathbf{G}_{\mathbf{x}}{ }^{*} \mathbf{I}\right)\right] .
$$

Here, $\mathbf{G}_{\mathbf{X}}$ is the Gabor filter matched to the dominant component at position $\mathrm{x}$. The operator $\otimes$ denotes correlation and * denotes convolution. The function $\varphi()$ weighs the contribution of the Gabor filter. For $\varphi()$, we use the formulation proposed in [41]

$$
\varphi(\xi)=-\left(1-\frac{1}{1+(|\xi| / k)^{2}}\right)
$$

where $k$ is a scaling constant. For example, in the case of fingerprint pattern generation, we can set $k$ according the desired contrast within the fingerprint ridges, as with (7).

The net effect of (9) is to produce a reaction where the pattern of specified granularity and directionality has not emerged. Therefore, (9) will stabilize when the local spectrum of I contains components within the localized spectral region covered by the Gabor filter frequency response. Since the patterns that emerge are not necessarily smooth, the simultaneous diffusion process allows the creation of smooth patterns localized in both space and frequency. 


\section{Rate of Reaction-Diffusion}

The update rate is an important factor effecting the implementation of reaction-diffusion for the repair of broken textures. In the previous sections, we specified the form for both reaction, $\mathbf{R}$, and diffusion, $\mathbf{D}$. We did not specify the two rate functions $\rho_{\mathrm{R}}$ and $\rho_{\mathrm{D}}$. Because the objectives of processing within the unoccluded region $\mathbf{U}$, the boundary region $\mathbf{B}$ and the occluded region $\mathbf{O}$ are different, we allow $\rho_{\mathrm{R}}(\mathbf{x})$ and $\rho_{\mathrm{D}}(\mathbf{x})$ to vary with position $\mathrm{x}$. Specifically, we diffuse within the entire unoccluded region $\mathbf{U}$, to provide simultaneous enhancement and disocclusion, and to balance the level of smoothing between the unoccluded and occluded regions. We also perform reaction within the boundary region $\mathbf{B}$, to guarantee pattern matching within the boundary region. The rate of reaction decreases within $\mathbf{B}$ as a function of distance from $\mathbf{O}$, the occluded/latent region.

Of course, both reaction and diffusion are performed within $\mathbf{O}$ at constant rates. For $\mathbf{x} \in \mathbf{O}$

$$
\rho_{\mathrm{R}}(\mathbf{x})=\rho_{\mathrm{RO}}
$$

which is determined by the constraints of stability and the maximum number of iterations. Likewise, if $\mathbf{x} \in \mathbf{O}$,

$$
\rho_{\mathrm{D}}(\mathbf{x})=\rho_{\mathrm{DO}}
$$

where $\rho_{\mathrm{DO}} \leq 1 / 4$ for stability.

For pixels outside of the occluded area $\mathbf{O}$, we have

$$
\rho_{\mathrm{D}}(\mathbf{x})=\rho_{\mathrm{DU}}
$$

where $\rho_{\mathrm{DU}}=\varepsilon \rho_{\mathrm{DO}}$. In the simulations given in Section $\mathrm{V}$, the value $\varepsilon=0.1$ was utilized. While we have not yet found an automatic method for selecting $\varepsilon$, two conflicting constraints need to be satisfied: the generated pattern should meld with the existing boundary of $\mathbf{B}$ while simultaneously inducing minimum distortion in the region $\mathbf{U}$.

If $\mathbf{x} \in \mathbf{U}$ but $\mathbf{x} \notin \mathbf{B}$, then $\rho_{\mathrm{R}}(x)=0$, since enforcing a pattern on the remainder of the image would produce distortion. However, if $\mathbf{x} \in \mathbf{B}$, we allow the rate of reaction to decrease to zero in a linear manner, as a function of the distance from the occluded region $\mathbf{O}$. Let $d(\mathbf{x}, \mathbf{O})$ specify the minimum Euclidean distance between point $\mathbf{x}$ and the occluded region $\mathbf{O}$. Then

$$
\rho_{\mathrm{R}}(\mathbf{x})=\rho_{\mathrm{RO}}\left[W_{\mathbf{B}}-d(\mathbf{x}, \mathbf{O})\right] / W_{\mathbf{B}}
$$

where $W_{\mathbf{B}}$ is the width of the band bordering the occluded region, defined as $W_{\mathbf{B}}=2 T_{\max }$. The reaction-diffusion algorithmic steps are illustrated in Fig. 4.

The success of the reaction-diffusion model depends on the reaction filters used in reconstructing the texture patterns. We utilize an AM-FM dominant component analysis to derive the filter parameters for the occluded regions, as described next.

\section{ESTIMATING THE DOMINANT COMPONENT MODUlATIONS}

In this section, we describe the AM-FM modeling of the input texture image and briefly review the dominant component analysis (DCA) technique [9], [10], [15] that is used for computing estimates of the dominant AM and FM functions along a contour that encloses the occluded or missing portion of the image.

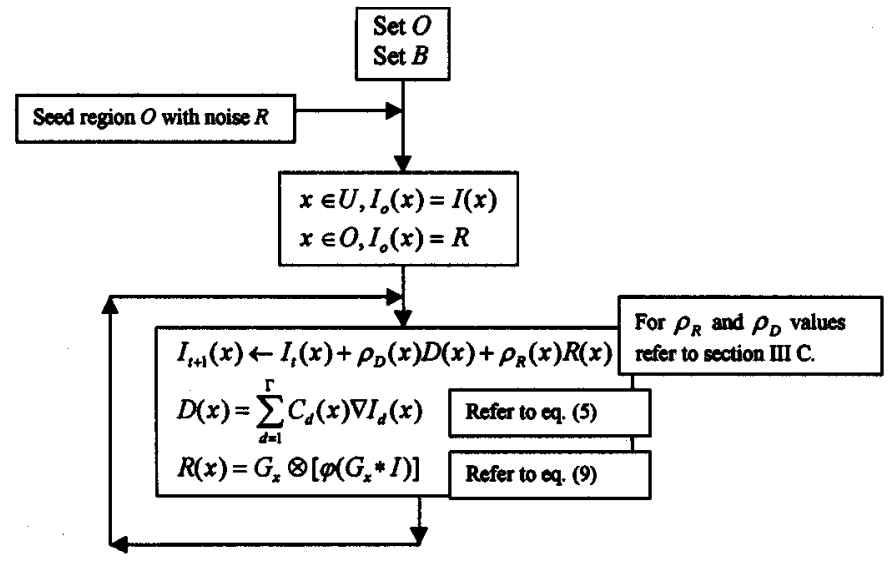

Fig. 4. Reaction-diffusion algorithm flowchart.

\section{A. AM-FM Modeling Fundamentals}

A 2-D AM-FM function $\mu(x, y)$ takes the form

$$
\mu(x, y)=a(x, y) \exp [j \psi(x, y)]
$$

where $a(x, y)$ and $\psi(x, y)$ are arbitrary real-valued functions. Without loss of generality, we assume that $a(x, y) \geq 0$. The AM and FM components of interest that are contained in $\mu(x, y)$ in (12) are the instantaneous amplitude $a(x, y)$ and the instantaneous frequency vector $\nabla \psi(x, y)=[u(x, y) v(x, y)]^{T}$. The functions $u(x, y)$ and $v(x, y)$ are the horizontal and vertical instantaneous frequencies of $\mu(x, y)$.

Given $\mu(x, y)$, the AM and FM functions may be calculated using the straightforward demodulation formulae

$$
\nabla \psi(x, y)=\operatorname{Re}\left[\frac{\nabla \mu(x, y)}{j \mu(x, y)}\right]
$$

and

$$
a(x, y)=|\mu(x, y)|
$$

which yield exact solutions at all points where $\mu(x, y) \neq 0$ [15] The frequency equation (13) may be interpreted as a specialized instance of a Poletti equation [34]; its use is motivated by the fact that the exponential function in (12) is invariant under differentiation.

Oriented, highly repetitive images such as fingerprints are well suited for AM-FM modeling because they are dominated by nonstationary, locally narrowband processes [6] and contain locally quasiperiodic patterns. However, note that $\mu(x, y)$ in (12) is complex-valued, whereas typical images are real-valued. The reason for considering a complex model is that the AM-FM functions of any real image $\Im(x, y)$ are not unique. By adding an imaginary component $j Q(x, y)$ to $\Im(x, y)$, we regularize the demodulation problem and obtain

$$
\mu(x, y)=\Im(x, y)+j Q(x, y)
$$

where $\Im(x, y)=a(x, y) \cos [\psi(x, y)]$ and $Q(x, y)=$ $a(x, y) \sin [\psi(x, y)]$.

Clearly, $\operatorname{Re}[\mu(x, y)]=\Im(x, y)$ irrespective of how we choose $Q(x, y)$. By setting $Q(x, y)$ equal to the directional multidimensional Hilbert transform of $\Im(x, y)$ [16], we ensure that $\mu(x, y)$ admits multidimensional analogs of many of the 
most attractive features of the well known 1-D analytic signal [8]. When $Q(x, y)$ is chosen in this way, we call $\mu(x, y)$ the analytic image associated with $\Im(x, y)$. The AM and FM functions of $\mu(x, y)$ can then be determined uniquely using (13) and (14), and we define these modulations to be the AM and FM functions of $\Im(x, y) .{ }^{1}$ The AM function $a(x, y)$ may be interpreted as the image contrast function, while $\nabla \psi(x, y)$ characterizes the local texture orientation and granularity.

\section{B. Discrete AM-FM Models}

A 2-D discrete version of the directional multidimensional Hilbert transform was given in [10], where it was specified in terms of its spectral multiplier. For a real-valued discrete image $I(x, y)$, this transform may be used to obtain an imaginary component $Q(x, y)$ and formulate the complex-valued AM-FM model

$$
\begin{aligned}
M(x, y) & =I(x, y)+j Q(x, y) \\
& =a(x, y) \exp [j \psi(x, y)] .
\end{aligned}
$$

We define $a(x, y)$ in (17) to be the AM function of $I(x, y)$.

Upon carefully discretizing (13) and (14), one obtains the equivalent expressions for the discrete demodulation algorithm:

$$
\begin{gathered}
|u(x, y)| \approx \arccos \left[\frac{M(x+1, y)+M(x-1, y)}{2 M(x, y)}\right] \\
\operatorname{sgn} u(x, y) \approx \operatorname{sgn} \arcsin \left[\frac{M(x+1, y)-M(x-1, y)}{2 j M(x, y)}\right] \\
|v(x, y)| \approx \arccos \left[\frac{M(x, y+1)+M(x, y-1)}{2 M(x, y)}\right] \\
\operatorname{sgn} v(x, y) \approx \operatorname{sgn} \arcsin \left[\frac{M(x, y+1)-M(x, y-1)}{2 j M(x, y)}\right]
\end{gathered}
$$

and

$$
a(x, y)=|M(x, y)|
$$

where $\nabla \psi(x, y)=[u(x, y) v(x, y)]^{T}$. While a derivation of these discrete frequency algorithms is based on rigorous theoretical arguments [15], some intuition can be gained by observing that the derivatives in (13) are replaced by first-order central differences and averages in (18)-(21). Unlike the continuous-domain frequency algorithm (13), which is exact, (18)-(21) are based on a novel discrete quasieigenfunction approximation and generally contain approximation errors unless $I(x, y)$ is a pure sinusoid [11]. However, these errors are typically negligible for images that are locally narrowband [15].

\footnotetext{
${ }^{1}$ Often, modulating functions similar to those calculated using the analytic image can alternatively be obtained by applying the multidimensional Teager-Kaiser operator [26] directly to a real-valued image. We generally prefer the approach based on the analytic image because of its strong theoretical correspondence to the 1-D analytic signal. For example, when the analytic image is used, the instantaneous and Fourier frequency spectra have identical first moments.
}

\section{Dominant Component Analysis}

For ideal digital fingerprint images and other oriented textural images, (18)-(22) can generally be used to compute the AM and FM functions accurately. Because of many factors [17], however, the images obtained in practice are often multipartite and fail to be everywhere locally narrowband [6], [14], [39]. These factors can lead to approximation errors in (18)-(21) that are not negligible. To overcome this problem, we model the textured image $I(x, y)$ not as the real part of a single AM-FM function, but rather as $I(x, y)=\operatorname{Re}[M(x, y)]$, where

$$
M(x, y)=\sum_{l=1}^{L} M_{l}(x, y)
$$

is the sum of $L$ AM-FM functions $M_{l}(x, y)=a_{l}(x, y) \exp \left[j \psi_{l}(x, y)\right]$. With this multicomponent model, $M(x, y)$ can still be obtained precisely as before [by setting $M(x, y)=I(x, y)+j Q(x, y)$ ] by virtue of the fact that the directional multidimensional Hilbert transform is a linear operator.

Our approach is then to demodulate all $L$ components in (23) simultaneously and select the AM and FM functions corresponding to the component that dominates the local image spectrum on a pixelwise basis. This technique is known as dominant component analysis (DCA) [9], [10], [15]. The single pair of $\mathrm{AM}$ and FM functions obtained by DCA are referred to as the dominant modulations of the image; they provide a rich description of the locally dominant image structure.

In performing DCA, the components are isolated from one another by analyzing $M(x, y)$ with a multiband Gabor filterbank of the type described in [2]. The choice of Gabor filters is motivated by two considerations. First, because of their optimal conjoint localization in space and frequency, an appropriately designed bank of Gabor filters is capable of resolving the components from one another spectrally, while simultaneously capturing spatially local nonstationarities. Second, the responses of Gabor filters are locally narrowband [1], [2].

Let $G_{i}(x, y)$ be the response of a particular Gabor filter (8) with impulse response $g_{i}(x, y)$ and frequency response $G_{i}(\omega)$. We assume that some particular component $M_{l}(x, y)$ dominates $G_{i}(x, y)$ at the pixel $(x, y)$, so that

$$
G_{i}(x, y)=M(x, y)^{*} g_{i}(x, y) \approx M_{l}(x, y)^{*} g_{i}(x, y) .
$$

By applying a sequence of quasieigenfunction approximations [3], [11], [15], one may verify the validity of estimating $\nabla \psi_{l}(x, y)$ by applying (18)-(21) directly to $G_{i}(x, y)$. The AM function $a_{l}(x, y)$ is then estimated using

$$
a_{l}(x, y) \approx\left|\frac{G_{i}(x, y)}{G_{i}\left[\nabla \psi_{l}(x, y)\right]}\right|
$$

which differs from (22) only in that the amplitude scaling of $G_{i}(x, y)$ relative to $M_{l}(x, y)$ has been factored out. Thus by applying (18)-(21) and (25) to the response of every filter in the filterbank, we obtain estimates of the AM and FM functions of all $L$ components in (23) at every pixel in the image. 
At each pixel, we define the dominant component as the one that dominates the response $G_{i}(x, y)$ of the filter that maximizes the selection criterion

$$
\Pi_{i}(x, y)=\frac{\left|G_{i}(x, y)\right|}{\max _{\omega}\left|G_{i}(\omega)\right|} .
$$

This criterion tends to select filters that are dominated by large amplitude components with instantaneous frequencies lying near the maximum transmission frequency of the filter, thereby rejecting cross-component interference and out-of-band noise. Estimates of the dominant modulations are taken from the filter that maximizes (26) on a pixelwise basis. The dominant frequency estimates along the perimeter of the occlusion are used to design the reaction filters for the reaction-diffusion process of (2). From the dominant component analysis, we obtain values for horizontal and vertical frequency, $u(x, y)$ and $v(x, y)$, at each image position. Recall that $\mathbf{B}$, the unoccluded region bordering the occluded region, is $2 T_{\max }$ pixels in width. We obtain a sample sequence of $u(x, y)$ values and $v(x, y)$ values contained within a path through $\mathbf{B}$ that is $T_{\max }$ pixels from $\mathbf{O}$. If the pattern is homogeneous in the occluded region, we can find the $(u, v)$ values used in (8) by taking the average $u(x, y)$ values and $v(x, y)$ values along the path.

In Section V, we demonstrate this approach, providing graphs of the dominant components around the occluded area. We also show example dominant component images obtained from this approach. The automation of the reaction filter selection is a major contribution of this work.

\section{RESULTS}

In this section, we present a set of results generated using the proposed reaction-diffusion model. The focal application we have chosen for demonstration is the repair of broken, occluded fingerprints. We also apply the AM-FM reaction-diffusion technique to a general set of textures. For the fingerprint application, the original images were collected from the NIST database. The occlusions are generated via natural [Fig. 7(a)] and synthetic means [Figs. 3(b), 5(a), 6(a)]. Results are given for the level line continuity disocclusion algorithm and also for the synthetic stripe formation process. Finally, a measure of accuracy is defined with respect to ground truth to show the fidelity of disocclusion process.

The analysis of fingerprint images has long attracted attention from the image processing community because of the many biometric and law enforcement applications. Beyond the significant work performed in coding and compression of fingerprint images, the processing techniques have mainly focused on enhancement or restoration of the fingerprint patterns from corrupted, noisy versions. A number of these fingerprint enhancement and restoration methods are guided by reaction-diffusion processes.

Because most latent fingerprints (those lifted from crime scenes) are fragmentary in nature, typical classification and file search methods are not practicable due to the occlusion of important features. Fingerprint occlusions can also occur through faulty mechanical operation in ink-based fingerprints. Focal points (deltas and cores) may be occluded if the finger

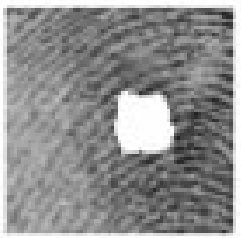

(a)

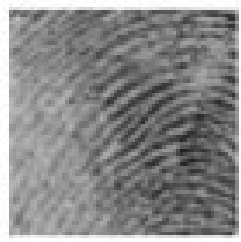

(b)

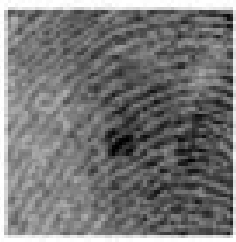

(c)
Fig. 5. (a) Occluded image of Fig. 2(a). (b) After disocclusion using proposed AM-FM reaction-diffusion method. (c) After disocclusion following stripe formation method.

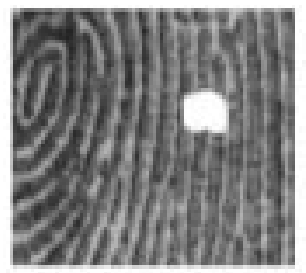

(a)

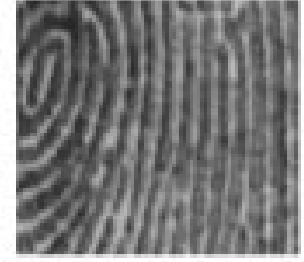

(b)
Fig. 6. (a) Occluded "stripe" fingerprint image of Fig. 3(a). (b) After disocclusion using AM-FM reaction-diffusion method.

has not been rolled from one side to the other, or if the bulb of the finger has not been completely inked. Similarly, if movement occurs, such as a twist or slip, the fingerprint can be partially smeared or blurred. Poor quality ink, excessive ink or runny ink can also obliterate important features such as the ridges. Finally, foreign substances and perspiration can also cause occlusion of features within the fingerprint. Image enhancement can be used to improve such prints, but the image enhancement procedure should not provide artifacts or false information. The enhancement process should allow for bifurcations, terminations, islands, and variation in ridge width. The PDE-based method suggested in this paper satisfies these needs, whereas continuity-based methods cannot recreate bifurcations, terminations, and islands. Typical texture repair approaches are not fit for prints with varying widths. Moreover, connecting broken ridges properly is a significant concern in ridge counting and tracing within the fingerprint analysis process. The reaction-diffusion solution given here adaptively connects the ridges across the occlusion, whereas traditional continuity-based solutions may lead to a false increase in ridges or leave the ridges unconnected across the occluded region.

Whether the occlusions result from latent prints or faulty printing on ink, the occlusions can adversely affect fingerprint classification and matching. The FBI divides these problems into three cases. In the first case, the impression is so scarred that neither the general type of pattern (arch, whorl, loop, etc.) nor the ridge tracing or counting can be accomplished. The second case includes prints in which the general type cannot be determined with reasonable accuracy, but ridges can be traced and counted. In the third case, the general type of fingerprint can be determined, but partial occlusion impedes ridge tracing and counting. Our disocclusion method concentrates on this third case-repairing occluded ridges for tracing and counting. Thus, the method described in this paper seeks to reconstruct occluded ridges. The approach, therefore, does not address the recreation of the fingerprint delta or the core. 


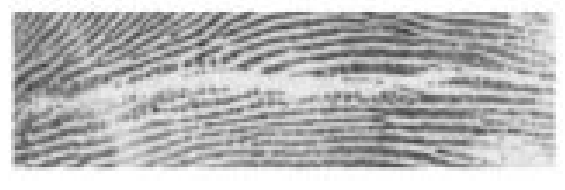

(a)

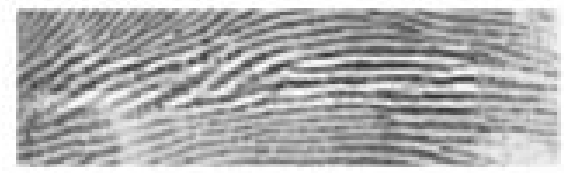

(b)

Fig. 7. (a) Original fingerprint image with "natural occlusion." (b) After disocclusion using AM-FM reaction-diffusion method.

Fig. 5(a) depicts a partially occluded image of the "oriented" fingerprint shown in Fig. 2(a). In Fig. 5(b), the disoccluded version of the image in Fig. 5(a) is shown, using the reaction-diffusion technique and DCA. Notice that the generated pattern is perceptually compatible with the overall embedded pattern of the fingerprint, providing a smooth transition at the boundary of the occlusion. The reconstructed image from the Meinhardt stripe formation process [30] is shown in Fig. 5(c). In the case of Fig. 5(c), for reconstruction, a smaller occluded patch is used compared to Fig. 5(a) since with the method of [30], larger occlusions are untenable. In all cases, note that the location of the occluded region is assumed to be known a priori. The AM-FM reaction-diffusion method does not address the occlusion detection problem.

Fig. 6(a) shows another fingerprint image with an arbitrarily selected missing patch from the original "stripe" fingerprint image of Fig. 3(a). Fig. 6(b) is the resultant image after disocclusion using reaction-diffusion. The preservation of the ridges in Fig. 6(b) is an example of the inter-region smoothing of anisotropic diffusion. Compare the result with that of Fig. 3(c) where a similar patch is reconstructed using level lines continuity. In the case of Fig. 3(c), the natural appearance of the reconstructed patch suffers due to enforcement of level line continuity.

Fig. 7(a) is a fingerprint image with natural but irregular occlusions due to partial contact of an uneven finger surface. The reconstructed image from reaction-diffusion is shown in Fig. 7(b). In this case, two Gabor filters were used to perform disocclusion. Note the junctions and bifurcations created in the texture regeneration. Also note that the thickness of the reconstructed ridges are almost uniform and compatible with the existing pattern.

As noted earlier, the successful pattern generation at the occlusion is rooted in the use of DCA in the bordering region of the occlusion. For the results cited above, the dominant component frequencies $(u, v)$ were plotted and are shown in Fig. 8. Fig. 8(a)-(c) are the corresponding dominant component frequencies of the occluded images of Figs. 5(a), 6(a) and 7(a) respectively. In Fig. 8, the $x$-axis represents the position of a path taken through $\mathbf{B}$ around the occluded region $\mathbf{O}$. The corresponding reconstructed images from the dominant component frequencies are shown in Figs. 9(a), (b), and (c), respectively. From Fig. 9, we can make two conclusions. First, the dominant component analysis extracts the dominant patterns in the fingerprint imagery. Second, dominant component analysis/reconstruction alone is not sufficient to perform disocclusion.

The quality of reconstruction is also substantiated using the mean square error (MSE) measure over the occluded region. In the case of Figs. 5(b) and 6(b), the original images shown in Figs. 2(a) and 3(a) are taken as ground truth. For Fig. 5(b) the
MSE is 755 for the method given here. Using the stripe formation process of [30], the MSE $\approx 5081$ in case of Fig. 5(c) (even using a smaller occlusion). Similarly, for Fig. 6(b), the MSE $\approx 335$ whereas the same MSE for Fig. 3(c) is 343.

As discussed in Section III, a boundary region B is considered for every occluded region $\mathbf{O}$. The rate of reaction-diffusion is decreased in region $\mathbf{B}$ in order to obtain a seamless integration of the generated pattern in the occluded region $\mathbf{O}$ within B. We have also extended the MSE measure to include region B. The MSE in the boundary region represents the amount of distortion the process has introduced in the existing pattern in order to have a perceptually acceptable oriented pattern generation. For Fig. 5(b), taking boundary region width to be ten pixels, the MSE is 99 , while taking the width to be 20 pixels, the MSE reduces to 51. For Fig. 6(b), under similar conditions, the MSE's are 182 and 79 respectively. The error decreases as the boundary region is increased in size and consequently distortion in the original pattern is minimized. For Fig. 5(c), the MSE's are 813 and 487, respectively, which are still greater in error than the AM-FM reaction-diffusion results.

Table I provides a summary of the example results. The table includes results for both fingerprint and general texture results. From the table, one may note the extent of the occluded region, the MSE for AM-FM reaction-diffusion result, the number of reaction-diffusion updates required, and the MSE for the level lines method result. Even though the level lines method is not appropriate for each example, we included results for this method for completeness. In seven of the eight examples, the AM-FM method provides superior results in terms of visual quality (see Figs. 10-13) and in terms of MSE. The final example (shown in Fig. 14) shows a case where the level lines approach is more appropriate than the AM-FM reaction-diffusion technique. The level lines method excels in the presence of smooth, directed textures without bifurcations or changes in orientation. In contrast, note the excelled performance of AM-FM reaction-diffusion on the "sand" image of Fig. 13. In this case, the AM-FM reaction-diffusion method is able to recreate the graininess and orientation of the original texture.

\section{CONCLUSIONS}

The paper presents a useful application of the reaction-diffusion paradigm. The major contribution is in developing a scheme that not only generates a texture for the missing part of an image but also maps and combines smoothly within the existing pattern. The approach does not use a continuity constraint on level lines [29], an interpolation scheme [19], nor a texture sewing technique [40]. The algorithm allows the intensity distribution of the generated pattern to be similar to that of the surrounding region. 


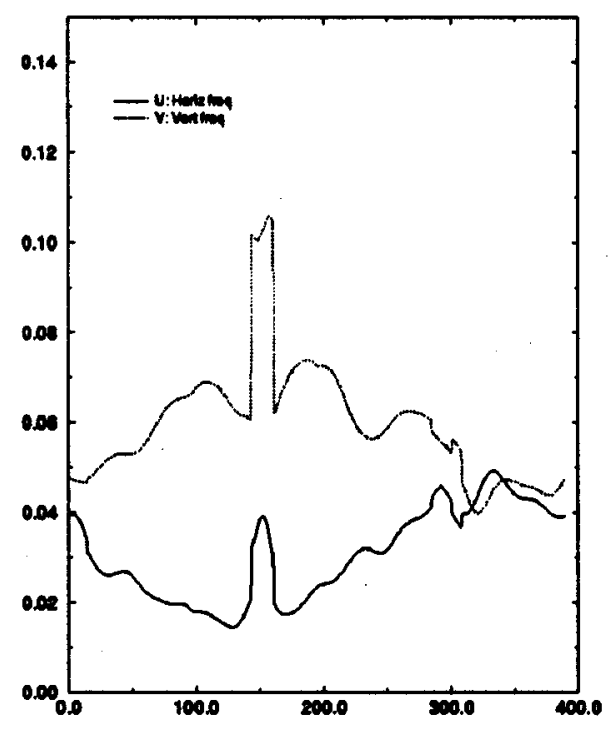

(a)

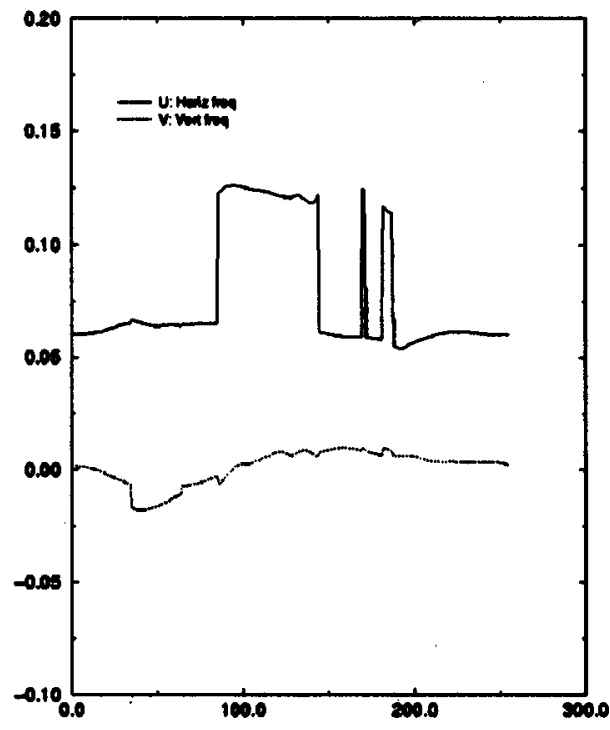

(b)

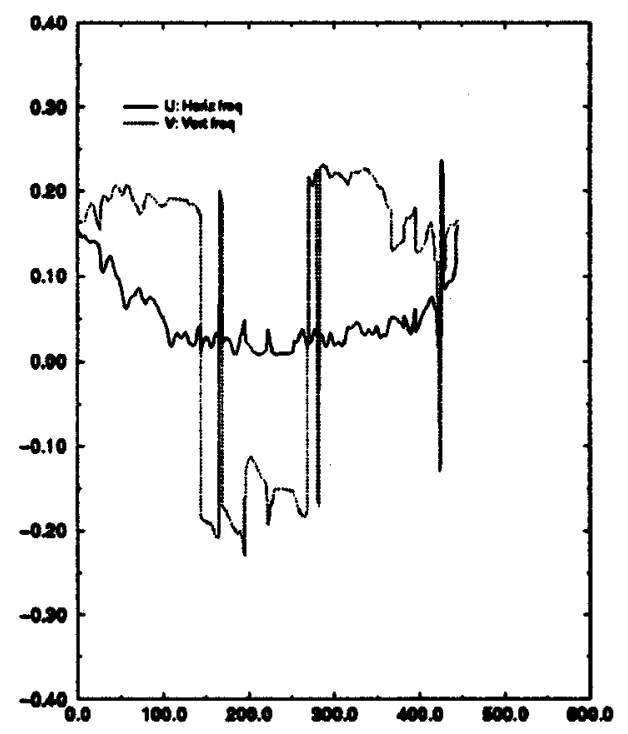

(c)

Fig. 8. Graphs of dominant component frequencies $(u, v)$ around the perimeter of the occluded area: (a) for "oriented" fingerprint image; (b) for "stripe" fingerprint image; and (c) for fingerprint image with "natural" occlusion. In each figure, the $x$-axis represents distance around the perimeter, and the $y$-axis represents frequency in cycles per sample.

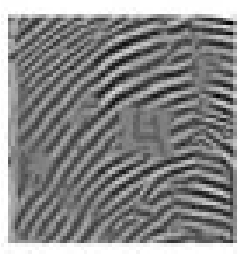

(a)

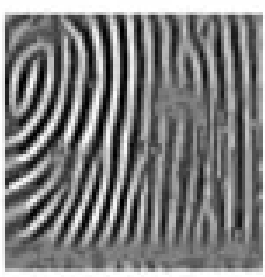

(b)

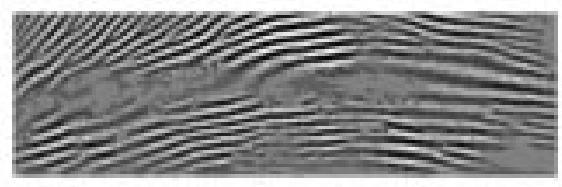

(c)

Fig. 9. Images generated from the occluded images by reconstructing the dominant components: (a) for "oriented" fingerprint image; (b) for "stripe" fingerprint image; and (c) for fingerprint image with "natural" occlusion.

In automating the selection of the texture parameters via dominant component analysis, we provide a rigorous method for tex- ture disocclusion. We apply the method to a significant practical problem, that of fingerprint repair/completion. 
TABLE I

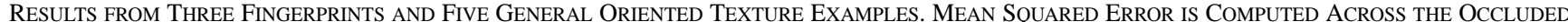
REGION 0 AND THE BOUNDARY REGION B

\begin{tabular}{l|l|l|l|l}
\hline Image & $\begin{array}{l}\text { Occluded } \\
\text { Region (top, } \\
\text { left) - } \\
\text { (bottom, } \\
\text { right) }\end{array}$ & $\begin{array}{l}\text { MSE } \\
\text { AM/FM } \\
\text { Reaction- } \\
\text { Diffusion } \\
\text { Method }\end{array}$ & $\begin{array}{l}\text { Iterations } \\
\text { For } \\
\text { Reaction- } \\
\text { Diffusion }\end{array}$ & $\begin{array}{l}\text { MSE } \\
\text { Level lines Method }\end{array}$ \\
\hline $\begin{array}{l}(50,50)- \\
(65,65)\end{array}$ & 755.1 & 32 & 1365.0 \\
\hline $\begin{array}{l}\text { Fingerprint } \\
\text { "Stripe" Fingerprint }\end{array}$ & $\begin{array}{l}(35,75)- \\
(55,95)\end{array}$ & 335.3 & 25 & 343.4 \\
\hline $\begin{array}{l}\text { Fingerprint with } \\
\text { "Natural Occlusion" }\end{array}$ & $\begin{array}{l}(25,0)- \\
(45,200)\end{array}$ & 2434.1 & 30 & 4867.8 \\
\hline Bark & $\begin{array}{l}(50,50)- \\
(65,65)\end{array}$ & 1651.6 & 25 & 2658.1 \\
\hline Woodgrain & $\begin{array}{l}(50,50)- \\
(65,65)\end{array}$ & 1764.5 & 50 & 2724.7 \\
\hline Rock & $\begin{array}{l}(50,50)- \\
(65,65)\end{array}$ & 98.8 & 10 & 164.5 \\
\hline Sand & $\begin{array}{l}(50,50)- \\
(65,65)\end{array}$ & 2273.5 & 25 & 3653.4 \\
\hline Wood & $\begin{array}{l}(55,65)- \\
(70,80)\end{array}$ & 3340.1 & 30 & 2088.5 \\
\hline
\end{tabular}

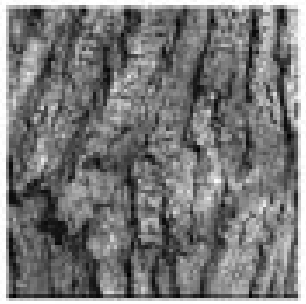

(a)

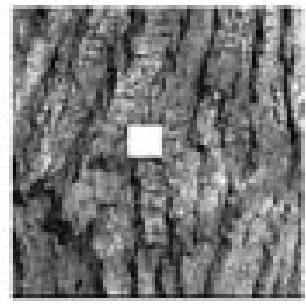

(b)

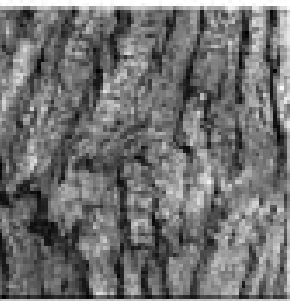

(c)

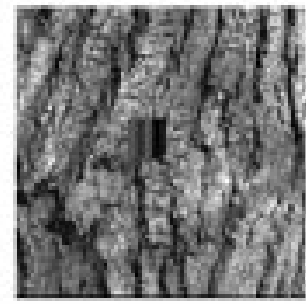

(d)

Fig. 10. (a) Original "bark" fingerprint image. (b) After occlusion (square region in black) in Fig. 3(a). (c) After disocclusion of Fig. 10(b) by AM-FM reaction-diffusion. (d) After disocclusion of Fig. 10(b) by the level line method.

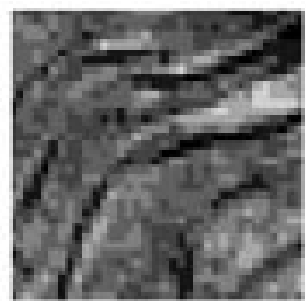

(a)

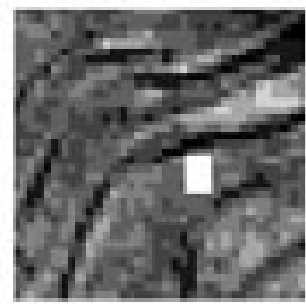

(b)

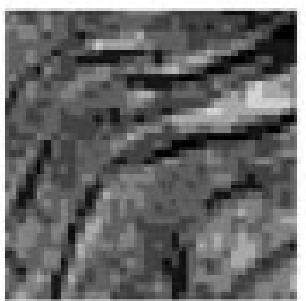

(c)

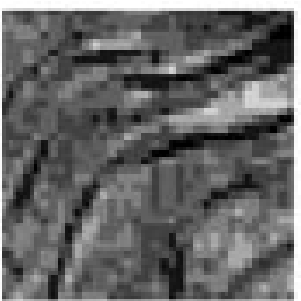

(d)

Fig. 11. (a) Original "woodgrain" fingerprint image. (b) After occlusion (square region in black) in Fig. 3(a). (c) After disocclusion of Fig. 11(b) by AM-FM reaction-diffusion. (d) After disocclusion of Fig. 11(b) by the level line method.

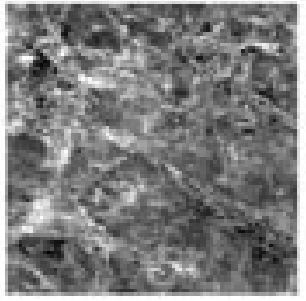

(a)

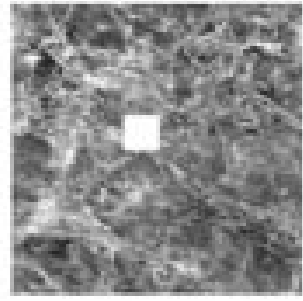

(b)

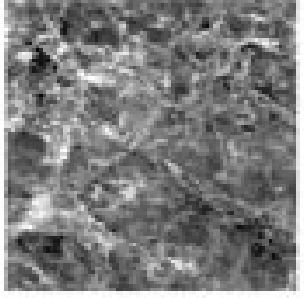

(c)

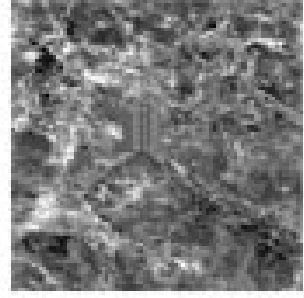

(d)

Fig. 12. (a) Original "rock" fingerprint image. (b) After occlusion (square region in black) in Fig. 3(a). (c) After disocclusion of Fig. 12(b) by AM-FM reaction-diffusion. (d) After disocclusion of Fig. 12(b) by the level line method. 


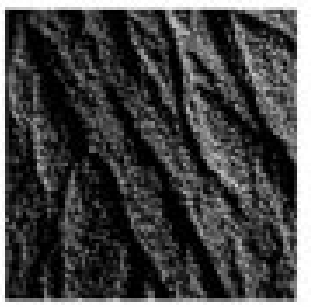

(a)

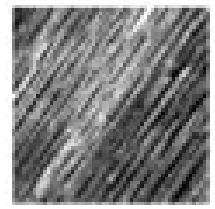

(a)

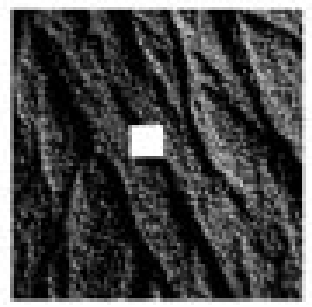

(b)

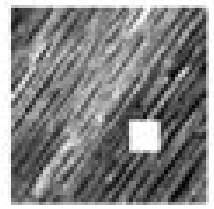

(b)

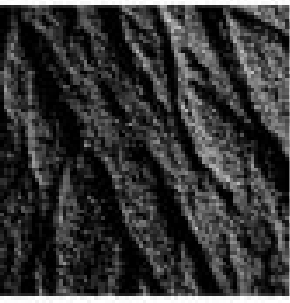

(c)

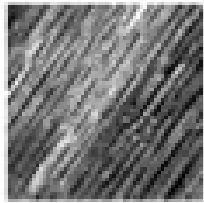

(c)

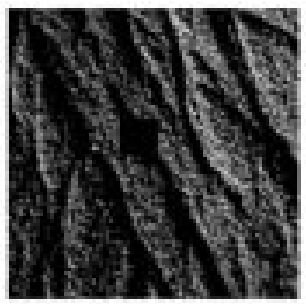

(d)

Fig. 14. (a) Original "wood" fingerprint image. (b) After occlusion (square region in black) in Fig. 3(a). (c) After disocclusion of Fig. 14(b) by AM-FM reaction-diffusion. (d) After disocclusion of Fig. 14(b) by the level line method.

\section{REFERENCES}

[1] A. C. Bovik, "Analysis of multichannel narrow-band filters for image texture segmentation," IEEE. Trans. Signal Processing, vol. 39, pp. 2025-2043, Sept. 1991.

[2] A. C. Bovik, M. Clark, and W. Geisler, "Multichannel texture analysis using localized spatial filters," IEEE Trans. Pattern Anal. Machine Intell., vol. 12, pp. 55-73, Jan. 1990.

[3] A. C. Bovik, J. P. Havlicek, D. S. Harding, and M. D. Desai, "Limits on discrete modulated signals," IEEE Trans. Signal Processing, vol. 45, pp 867-879, Apr. 1997.

[4] A. C. Bovik, P. Maragos, and T. F. Quatieri, "AM-FM energy detection and separation in noise using multiband energy operators," IEEE. Trans. Signal Processing, vol. 41, pp. 3245-3265, Dec. 1993.

[5] F. Catte, P.-L. Lions, J.-M. Morel, and T. Coll, "Image selective smoothing and edge detection by nonlinear diffusion," SIAM J. Numer. Anal., vol. 29, pp. 182-193, 1992.

[6] L. Cohen, "What is a multicomponent signal," in Proc. IEEE Int. Conf. Acoustics, Speech, Signal Processing, vol. V, San Francisco, CA, Mar. 1992, pp. 113-116.

[7] A. A. Efros and T. K. Leung, "Texture synthesis by nonparametric sampling," in Proc. IEEE Int. Conf. Computer Vision (ICCV'99), 1999.

[8] D. Gabor, "Theory of communication," J. Inst. Elect. Eng., vol. 93, no. III, pp. 429-457, 1946.

[9] J. P. Havlicek, "The evolution of modern texture processing," Elektrik, Turkish J. Elect. Eng. Comput. Sci., vol. 5, no. 1, pp. 1-28, 1997.

[10] J. P. Havlicek and A. C. Bovik, "Modulation models," in The Image and Video Processing Handbook, A. C. Bovik, Ed. New York: Academic, 1999.

[11] J. P. Havlicek, D. S. Harding, and A. C. Bovik, "Discrete quasieigenfunction approximation for AM-FM image analysis," in Proc. IEEE Int. Conf. Image Processing, Lausanne, Switzerland, Sept. 1996, pp. 633-636.

[12] J. P. Havlicek, J. W. Havlicek, and A. C. Bovik, "The analytic image," in Proc. IEEE Int. Conf. Image Processing, Santa Barbara, CA, Oct. 1997.

[13] J. P. Havlicek, D. S. Harding, and A. C. Bovik, "The multi-componen AM-FM image representation," IEEE Trans. Image Processing, vol. 5, pp. 1094-1100, June 1996

[14] — "Multicomponent multidimensional signals," Multidimen. Syst. Signal Process., vol. 9, no. 4, pp. 391-398, Oct. 1998.

[15] — , "Multidimensional quasieigenfunction approximations and multicomponent AM-FM models," IEEE Trans. Image Processing, to be published.

[16] J. P. Havlicek, J. W. Havlicek, N. D. Mamuya, and A. C. Bovik, "Skewed 2D Hilbert transforms and computed AM-FM models," in Proc. IEEE Int. Conf. Image Processing, Chicago, IL, Oct. 1998.

[17] L. Hong, Y. Wan, and A. Jain, "Fingerprint image enhancement: Algorithm performance and evaluation," IEEE. Trans. Pattern Anal. Machine Intell., vol. 20, pp. 777-789, Aug. 1998.

[18] G. Kanizsa, Organization in Vision. New York: Praeger, 1979.
[19] M. Kass and A. Witkin, "Analyzing oriented patterns," Comput. Vis. Graph., Image Process., vol. 37, pp. 362-385, 1987.

[20] A. Kokaram, Motion Picture Restoration: Digital Algorithms for Artefact Suppression in Degraded Motion Picture Film and Video. Berlin, Germany: Springer-Verlag, 1998.

[21] A. Kokaram and S. J. Godsill, "A system for reconstruction of missing data in image sequences using sampled 3D AR models and MRF motion priors," in Proc. ECCV, April 1996, pp. 613-624.

[22] A. Kokaram, R. D. Morris, W. J. Fitzgerald, and P. J. Rayner, "Detection of missing data image sequences," IEEE Trans. Image Processing, vol. 4, pp. 1496-1508, 1995.

[23] - "Interpolation of missing data in image sequences," IEEE Trans. Image Processing, vol. 4, pp. 1509-1519, 1995.

[24] P. Kornprobst, R. Deriche, and G. Aubert, "Nonlinear operators in image restoration," Proc. CVPR, pp. 325-331, 1997.

[25] S. Lu and P. C. Doerschuk, "Demodulators for AM-FM models of speech signals: A comparison," in Proc. IEEE Int. Conf. Acoustics, Speech, Signal Processing, Atlanta, GA, May 1996, pp. 263-266.

[26] P. Maragos and A. C. Bovik, "Image demodulation using multidimensional energy separation," J. Opt. Soc. Amer. A, vol. 12, no. 9, pp. 1867-1876, Sept. 1995

[27] P. Maragos, J. F. Kaiser, and T. F. Quatieri, "On amplitude and frequency demodulation using energy operators," IEEE Trans. Signal Processing, vol. 41, pp. 1532-1550, Apr. 1993.

[28] D. Marr and E. Hildreth, "Theory of edge detection," in Proc. R. Soc. Lond. B, vol. 275, 1976, pp. 484-519.

[29] S. Masnou and J. Morel, "Level lines based disocclusion," in Proc. IEEE Int. Conf. Image Processing, Chicago, IL, 1998.

[30] H. Meinhardt, Models of Biological Pattern Formation. New York: Academic, 1982.

[31] M. Nitzberg, D. Mumford, and T. Shiota, Filtering, Segmentation and Depth. Berlin, Germany: Springer-Verlag, 1993, vol. 662.

[32] N. Paragios and R. Deriche, "Geodesic active regions for supervised texture segmentation," INRIA, Sophia-Antipolis, France, INRIA Res. Rep. RR-3440, 1999.

[33] P. Perona and J. Malik, "Scale-space and edge detection using anisotropic diffusion," IEEE Trans. Pattern Anal. Machine Intell., vol. 12, pp. 629-639, 1990.

[34] M. Poletti, "Instantaneous frequency and conditional moments in the time-frequency plane," IEEE Trans. Signal Processing, vol. 39, pp. 755-756, Mar. 1991.

[35] C. Price, P. Wambacq, and A. Oosterlinck, "Image enhancement and analysis with reaction-diffusion paradigm," Proc. Inst. Elect. Eng., pt. 1, vol. 137, no. 3, pp. 136-144, June 1990.

[36] C. A. Segall and S. T. Acton, "Morphological anisotropic diffusion," in Proc. IEEE Int. Conf. Image Processing, Santa Barbara, CA, Oct. 26-29, 1997.

[37] A. Sherstinsky and R. Picard, " $M$-Lattice: From morphogenesis to image processing," IEEE Trans. Image Processing, vol. 5, pp. 1137-1150, July 1996. 
[38] A. Turing, "The chemical basis of morphogenesis," Phil. Trans. R. Soc. $B$, vol. 237, pp. 37-72, 1952 .

[39] D. Wei and A. C. Bovik, "On the instantaneous frequencies of multicomponent AM-FM signals," IEEE Signal Processing Lett., vol. 5, pp. 84-86, Apr. 1998.

[40] A. Witkin and M. Kass, "Reaction-diffusion textures," Comput. Graph., vol. 25, pp. 299-306, July 1991.

[41] S. Zhu and D. Mumford, "Prior learning and Gibbs reaction-diffusion," IEEE Trans. Pattern Anal. Machine Intell., vol. 19, pp. 1236-1250, Nov. 1997.

[42] S. C. Zhu, Y. Wu, and D. Mumford, "Filters, random fields and maximum entropy," Int. J. Comput. Vis., vol. 27, pp. 1-20, 1998.

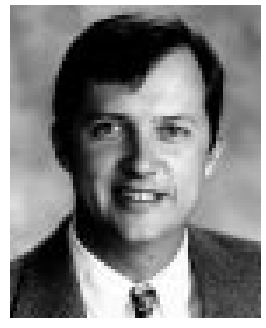

Scott T. Acton (S'89-M'93-SM'99) received the B.S. degree in electrical engineering from Virginia Tech, Blacksburg, in 1988 as a Virginia Scholar. He received the M.S. degree in electrical engineering and the Ph.D. degree in electrical engineering from the University of Texas at Austin in 1990 and 1993, respectively.

$\mathrm{He}$ is currently an Associate Professor with the Department of Electrical and Computer Engineering, University of Virginia, Charlottesville. He has worked in industry for AT\&T, the MITRE Corporation, and Motorola, Inc., and in academia for Oklahoma State University, Stillwater. His research interests include biomedical image analysis, multiscale signal representations, diffusion algorithms, active contours, video tracking, area morphology, image segmentation, and content-based retrieval.

Dr. Acton is the winner of the 1996 Eta Kappa Nu Outstanding Young Electrical Engineer Award, a national award that has been given annually since 1936. He also received the 1997 Halliburton Outstanding Young Faculty Award. He serves as Associate Editor of the IEEE TRANSACTIONS ON IMAGE PROCESSING and is an active participant in the IEEE, SPIE, and Eta Kappa Nu.

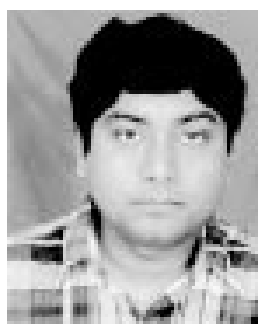

Dipti Prasad Mukherjee received the B.E. degree from Jadavpur University, Calcutta, India, in 1985, the M.S. degree from the University of Saskatchewan, Saskatoon, SK, Canada, in 1989, and the Ph.D. degree from Indian Statistical Institute (ISI), Calcutta, in 1996

$\mathrm{He}$ is currently a Faculty Member with the Electronics and Communication Sciences Unit, ISI. He was a Visiting Assistant Professor in Oklahoma Imaging Laboratory, School of Electrical and Computer Engineering, Oklahoma State University, Stillwater, in 1998-1999. He was a UNDP Fellow with the Robotics Research Group, University of Oxford, Oxford, U.K., in 1992. His research interests are in the areas of computer vision and graphics. He has published 25 peer-reviewed journal papers and is the author of a textbook on computer graphics and multimedia.

Dr. Mukherjee was the recipient of UNESCO-CIMPA Fellowships to INRIA, France, in 1991, 1993, and 1995 and a Fellowship to ICTP, Trieste, Italy in 2000.

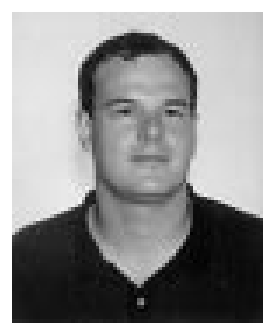

Joebob P. Havlicek (S'84-M'88-SM'98) was born in West Lafayette, IN, on April 7, 1964. He received the B.S. degree in 1986 and the M.S. degree in 1988 from Virginia Tech, Blacksburg, and the Ph.D. degree in 1996 from the University of Texas at Austin, all in electrical engineering.

From 1984 to 1987, he was a Software Engineer with Management Systems Laboratories, Blacksburg. From 1987 to 1989 , he was affiliated with SFA, Inc., Landover, MD, and from 1987 to 1997 he was with the Naval Research Laboratory, Washington, DC, where his work included high-performance signal and image processing. In 1993, he was a Programmer-Analyst with Ralph Kirkley Associates, Inc., Austin, TX, working on-site in the Multimedia Division, IBM, Austin. Since January 1997 he has been an Assistant Professor with the School of Electrical and Computer Engineering, University of Oklahoma, Norman. His research interests include signal and image processing, multimedia communications, and intelligent transportation systems.

Dr. Havlicek was recipient of the University of Oklahoma IEEE Favorite Instructor Award in 1998 and 2000, the Department of the Navy Award of Merit for Group Achievement in 1990, and the 1992 University of Texas Engineering Foundation Award for Exemplary Engineering Teaching while Pursuing a Graduate Degree. He is a member of Tau Beta Pi, Phi Kappa Phi, and Eta Kappa Nu.

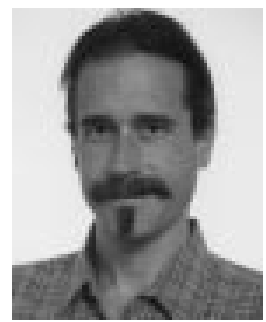

Alan Conrad Bovik (S'80-M'80-SM'89-F'96) received the $\mathrm{Ph} . \mathrm{D}$. degree in electrical and computer engineering in 1984 from the University of Illinois, Urbana-Champaign.

$\mathrm{He}$ is currently the Robert Parker Centennial Endowed Professor in the Department of Electrical and Computer Engineering at the University of Texas at Austin, where he is the Associate Director of the Center for Vision and Image Sciences. During the Spring of 1992, he held a visiting position in the Division of Applied Sciences, Harvard University, Cambridge, MA. His current research interests include digital video, image processing and computational modeling of biological visual perception. He has published more 300 technical articles in these areas and holds two U.S. patents. $\mathrm{He}$ is also the editor/author of the Handbook of Image and Video Processing (New York: Academic, 2000).

Dr. Bovik was named Distinguished Lecturer of the IEEE Signal Processing Society in 2000, received the IEEE Signal Processing Society Meritorious Service Award in 1998, the IEEE Third Millennium Medal in 2000, the University of Texas Engineering Foundation Halliburton Award and is a two-time Honorable Mention winner of the International Pattern Recognition Society Award for Outstanding Contribution (1988 and 1993). He has been involved in numerous professional society activities, including, currently: Editor-in Chief, IEEE TRansactions on Image Processing and Editorial Board, PROCEEDINGS OF THE IEEE. He also serves on the editorial boards of several other technical journals and on several international technical committees. He was the Founding General Chairman of the First IEEE International Conference on Image Processing, held in Austin, TX, in November, 1994. He is a Registered Professional Engineer in the State of Texas and is a frequent consultant to industry and academic institutions. 